FORMATION Formation emploi

Revue française de sciences sociales

121 | janvier-mars 2013

L'emploi public : nouveau système de règles, nouvelles stratégies d'acteurs

\title{
« Moderniser » les pratiques d'évaluation du travail dans la fonction publique : Analyse exploratoire du cas d'un hôpital public
}

Modernizing assessment practices of work in the civil service: An exploratory study of a French public hospital

"Modernisierung" der Methoden für die Beurteilung der Arbeit im öffentlichen Dienst - Analytische Untersuchung des Fallbeispiels öffentliches Krankenhaus "Modernizar" las prácticas de evaluación del trabajo en la función pública. Análisis exploratorio del caso de un hospital público

\section{Sandra Bertézène et Benjamin Dubrion}

\section{OpenEdition Journals}

Édition électronique

URL : http://journals.openedition.org/formationemploi/3935

DOI : 10.4000/formationemploi.3935

ISSN : 2107-0946

Éditeur

La Documentation française

Édition imprimée

Date de publication : 15 février 2013

Pagination : 83-105

ISSN : 0759-6340

Référence électronique

Sandra Bertézène et Benjamin Dubrion, « « Moderniser » les pratiques d'évaluation du travail dans la fonction publique : Analyse exploratoire du cas d'un hôpital public », Formation emploi [En ligne], 121 | janvier-mars 2013, mis en ligne le 15 février 2015, consulté le 30 octobre 2020. URL : http://

journals.openedition.org/formationemploi/3935; DOI : https://doi.org/10.4000/formationemploi.3935 


\title{
«Moderniser » les pratiques d'évaluation du travail dans la fonction publique
}

\section{Analyse exploratoire du cas d'un hôpital public}

\begin{abstract}
BenJamin Dubrion ${ }^{1}$
maître de conférences en économie à Sciences Po Lyon, membre du laboratoire Triangle CNRS

UMR 5206.

SANDRA BERTÉŻ̇ne

maître de conférences en sciences de gestion à l'université Claude Bernard Lyon 1, membre du laboratoire SAF (Sciences actuarielles et financières) (EA 2429).
\end{abstract}

Résumé

« Moderniser » les pratiques d'évaluation du travail dans la fonction publique. Analyse exploratoire du cas d'un hôpital public

Cet article s'appuie sur une étude exploratoire menée dans un hôpital public. Il étudie la mise en place d'outils d'évaluation du travail classiquement utilisés dans les grandes organisations privées qui visent une plus grande prise en compte de la contribution individuelle des agents. Dans une démarche compréhensive, et à la lumière des travaux de James March, il apparaît que ces " outils de gestion " conduisent les acteurs, surtout les cadres de soins, à modifier leur façon d'être et de concevoir leur activité. Leur représentation du «bon travail » semble alors être mise en tension.

Mots clés : Fonction publique hospitalière, établissement hospitalier, évaluation, modernisation

Abstract

Modernizing assessment practices of work in the civil service An exploratory study of a French public hospital

Based on an exploratory research in a French public hospital, this paper studies the effects of work assessment tools that are generally used in firms in order to evaluate the individual contribution of employees to the organization. In a comprehensive perspective and in the light of the works of James March on decision making, we show that these tools lead

1. Nous tenons à remercier la rédaction de la revue et les rapporteurs anonymes pour leurs remarques et suggestions visant à améliorer ce texte. Que Bernard Baudry, Hervé Charmettant et Virgile Chassagnon soient également remerciés pour leurs lectures sur des versions antérieures de ce travail. 
health middle managers to change their behavior and perception of their activity. They destabilize their representation of what a "good work" is.

Key words: Civil service in hospitals, hospital, evaluation, modernization

Journal of economic literature: H 83, I19, M 54

Traduction : Auteurs

En France, la thématique de l'évaluation du travail fait l'objet de nombreux débats ces dernières années ${ }^{2}$. Les discussions sont surtout apparues avec le développement, à partir des années 90 au sein des organisations privées, de pratiques de gestion des ressources humaines (GRH) focalisées sur la prise en compte des performances et compétences individuelles (Baudry et Dubrion, 2005). Plus récemment, les réformes touchant la fonction publique dans les années 2000 - LOLF (Loi organique relative aux lois de finances) et RGPP (Révision générale des politiques publiques) ${ }^{3}$ notamment - et qui ont fait évoluer la gestion publique des ressources humaines, prolongent aujourd'hui les débats et discussions sur cette thématique. Ainsi à cet égard, certains spécialistes en management public parlent depuis peu de GRH publique "modernisée ", GRH dont justement "la principale transformation concerne la mise en place d'entretiens individuels [...] et de procédures de concertation sur les objectifs et moyens entre hiérarchiques et subordonnés" (Bartoli, 2009, p. 162).

Cet article s'inscrit dans la lignée des interrogations soulevées par le développement des pratiques d'évaluation du travail focalisées sur les individus et qui se diffusent ces dernières années dans la fonction publique. Nous nous centrons ici sur la fonction publique hospitalière. Plus précisément, nous examinons comment les dispositifs d'évaluation du travail orientés vers la recherche d'une plus grande prise en compte de la contribution individuelle des agents publics à l'organisation concourent à bousculer les valeurs qui sous-tendent leurs actions. À cet effet, nous étudions le cas d'un hôpital public ( $c f$. encadré $\mathbf{1})$.

2. Le lecteur pourra notamment consulter les dossiers spéciaux ou tribunes consacrés à ce thème par plusieurs revues académiques comme la Nouvelle revue de psychosociologie (2009), les Cahiers internationaux de sociologie (2010), la «tribune » de Vatin et alii (2010) dans la Revue Française de Gestion, Actes de la recherche en sciences sociales (2011) ou encore Travailler (2011).

3. La LOLF et la RGPP sont des dispositifs mis en place par l'État, au cours des années 2000, en France, afin d'améliorer l'action publique. 


\section{Encadré 1: Méthodologie}

Ce travail fait suite à une intervention menée en 2010 dans un hôpital public. La DRH souhaitait diagnostiquer l'effet de l'instauration, auprès des cadres de soins, d'une nouvelle grille d'évaluation du travail. Elle visait à mettre en œuvre une politique de gestion prévisionnelle des métiers et des compétences (GPMC). A cet effet, en 2009, une étude y avait été menée en interne Elle s'était traduite par la construction d'un répertoire des métiers internes à l'établissement puis par une première analyse quantitative et qualitative des métiers et des effectifs ainsi que de leur évolution anticipée à cinq ans.

L'hôpital public étudié est situé dans une ville de province. Il est de petite taille, avec un peu moins de 500 lits. Il intègre un établissement d'hébergement pour personnes âgées dépendantes.

Du point de vue méthodologique, le travail mené ici s'inscrit dans une approche qualitative soit un " ensemble de techniques interprétatives qui visent à décrire, décoder, traduire, et plus généralement à entrer en accord avec la signification, et non la fréquence, de certains phénomènes sociaux plus ou moins naturels » (Van Maanen, 1979, p. 520). Suivant une opposition fondamentale en sciences sociales (Aron, 1981), la démarche adoptée n'est pas tant explicative que compréhensive. Elle ne vise pas en effet prioritairement à valider des hypothèses posées au départ par le chercheur et à étudier les relations de causalités existantes entre différentes variables. Il s'agit plutôt de comprendre le sens des actions des agents en situation, de questionner la rationalité de leurs actions. Dans cette voie reposant sur les observations d'un terrain d'étude, le chercheur donne un sens au réel, fournit des interprétations de la réalité étudiée à travers ce qu'en disent les acteurs (Wacheux, 1996).

Le travail de terrain que nous avons mené repose sur deux sources principales d'information :

(1) Des entretiens semi-directifs. Ils ont permis d'accéder aux représentations et aux interprétations des situations connues par les acteurs (Miles et Huberman, 1991). Vingt-trois personnes ont été interrogées : deux membres de la direction, six cadres de santé ou faisant fonction, deux médecins, treize personnels sans responsabilité hiérarchique (secrétaire médicale, infirmiers diplômés d'État, aides-soignants, agents de service hospitalier et agents d'entretien).

Notre guide d'entretien était composé de trois parties renvoyant à trois grands thèmes : la politique de management des ressources humaines, l'évaluation individuelle, les outils et dispositifs de management des ressources humaines. Pour être plus précis, la thématique de l'évaluation du travail, centrale dans notre travail, était décomposée en trois sous-thèmes abordant (a) ce qui est attendu et comment cela est perçu/vécu ; (b) ce qui se fait et comment cela est perçu/vécu ; (c) les décalages existant entre ces deux éléments.

Le recueil et l'analyse des données ont été réalisés en suivant la méthode préconisée par l'approche socio-économique (Savall et Zardet, 2004). Les verbatim issus des entretiens ont été dépouillés et exploités afin d'en extraire des phrases-témoins. Celles-ci ont été, par la suite, retraitées par thèmes, sous-thèmes et idées-clés. L'idée-clé est une idée générique pouvant rassembler plusieurs illustrations d'une même pratique. Certaines phrases-témoins recueillies lors des entretiens, sans modification, ont été reprises de manière anonyme dans le présent article.

(2) L'autre source d'information est de nature formelle : elle comprend un ensemble de documents internes et externes à l'hôpital qui renseignent sur le mode de management mis en place par la direction, et plus spécifiquement la direction des ressources humaines : les outils d'évaluation bien évidemment (grilles d'évaluation, grilles de compétences, descriptifs d'activités, fiches de postes, etc.), des études menées sur la GPMC, des synthèses portant sur les formations suivies par les agents, des documents administratifs de type circulaire. 
Par évaluation du travail, nous entendons l'action de déterminer la qualité et la valeur du travail réalisé par un individu. Cette action s'appuie sur un ensemble de règles formelles que nous appréhendons comme des « outils de gestion ». Dans le prolongement des travaux initiés en gestion par des auteurs comme Berry (1983) ou Moisdon (1997), nous nous interrogeons sur le rôle et les effets de l'instrumentation gestionnaire (Gilbert, 1998) dans la conduite des organisations; plus spécifiquement sur les outils d'évaluation qui ont au départ été conçus pour évaluer le travail des agents des organisations privées et qui sont aujourd'hui de plus en plus utilisés au sein des organisations publiques, sous l'effet des réformes de la fonction publique.

Sur la base de notre étude exploratoire, nous postulons que les outils d'évaluation participent de la construction de rôles sociaux chez les membres de l'organisation. Ils les conduisent en outre à modifier leur façon d'être et de concevoir leur activité dans un sens qui peut aller à l'encontre de la représentation qu'ils se font d'un "bon travail ". Nous montrons que les cadres de soins, qui sont au cœur des réformes récentes touchant le travail public hospitalier, sont bousculés par le développement des outils d'évaluation individualisée.

Nous procédons en trois temps. Il s'agit d'abord de présenter le contexte général de réforme de l'hôpital public, en France, puis ses prolongements en matière de GRH. Nous analysons ensuite les effets de la mise en œuvre d'une nouvelle grille d'évaluation du travail dans l'hôpital étudié, en soulignant en quoi le nouvel outil déstabilise les représentations des acteurs de l'évaluation, surtout des cadres. Enfin, nous tentons de donner sens aux évolutions constatées, à la lumière d'une grille d'analyse constructiviste des outils de gestion ; à cet effet, nous nous appuyons notamment sur les travaux de James March sur la prise de décision en organisation, que nous appliquons au jugement porté sur la valeur du travail. Nous nous questionnons alors sur certaines voies visant à atténuer les tensions vécues par les acteurs suite à l'introduction de ce "nouveau » type d'outils d'évaluation.

\section{De la « modernisation » à l'hôpital ou le renforcement de la logique gestionnaire}

Les évolutions rencontrées dans l'hôpital étudié, en matière d'évaluation du travail, doivent être comprises au regard d'un contexte de "modernisation " du fonctionnement de l'hôpital public qui s'est accéléré à partir des années 1990, impactant alors la gestion des ressources humaines.

\subsection{Les trois grands principes de la « modernisation » de l'hôpital public}

Ces vingt dernières années ont vu se renforcer l'application de la logique gestionnaire à l'hôpital (Moisdon et Tonneau 1999). Plusieurs réformes ont structuré les transformations du service public hospitalier avec un double objectif : améliorer la qualité des 
soins dispensés au sein des établissements et maitriser l'évolution des dépenses de santé. Les dispositifs mis en œuvre pour traiter aux mieux ces deux questions s'appuient sur un ensemble de principes issus du Nouveau Management Public et qui se sont affirmés à l'hôpital depuis les années 90 .

Un des principes fondamentaux structurant les réformes hospitalières récentes est celui de la contractualisation. Les procédures de contractualisation ont été introduites avec la loi du 31 juillet 1991. Elle prévoit la possibilité, pour les établissements hospitaliers, de contractualiser avec l'État ou l'assurance maladie sur un ensemble d'objectifs et de moyens. Cette démarche de contractualisation a été systématisée avec les réformes suivantes, la contractualisation se faisant dès lors de façon externe et interne aux établissements. La loi HPST (Hôpital, patients, santé et territoire) de 2009 consacre ainsi une contractualisation à trois niveaux - État/Agence régionale de santé (ARS), ARS/ direction d'établissement, puis direction d'établissement/pôle d'activité - chaque étape spécifiant des objectifs attendus appréhendés par des indicateurs quantifiables (Couty et alii, 2009).

L'expansion de cette logique de contractualisation s'est opérée de concert avec la délégation de gestion devant permettre une meilleure allocation des ressources. $\mathrm{La}$ délégation de gestion prend surtout corps avec la création des Agences régionales d'hospitalisation (ARH) en 1996, devenues ARS par la suite. Couplée à la contractualisation, elle vise une responsabilisation accrue des établissements (Cueille et Renucci, 2000) ; cette responsabilisation étant étendue au niveau des acteurs et ayant des effets sur les pratiques de gestion des ressources humaines suite à la mise en œuvre de la "nouvelle gouvernance " et la création des pôles d'activité du Plan Hôpital 2007 (Crozet et alii, 2008). L'instauration de la tarification à l'activité, à partir de 2004, puis la loi HPST accompagnent ce mouvement. Il s'agit de responsabiliser l'ensemble des acteurs de la ligne hiérarchique allant de l'État aux pôles d'activités.

Enfin, le principe de certification (anciennement nommée accréditation) est un principe fort traversant lui aussi les réformes récentes de l'hôpital public. Son origine remonte à des réflexions menées dans les années 80 sur la problématique de la qualité à l'hôpital (Minvielle, 2003). L'évaluation de la qualité des soins est rendue obligatoire avec la loi de 1991. Elle a été systématisée par l'Agence nationale d'accréditation et d'évaluation en santé (ANAES) créée en 1996, remplacée par la Haute autorité de la santé (HAS). Celle-ci, depuis 2004, a notamment pour objectif de coordonner la mise en œuvre de la certification des établissements de santé, l'accréditation des médecins et des équipes médicales, et l'évaluation des pratiques professionnelles.

Les développements de la contractualisation, de la délégation de gestion et de la certification constituent autant d'éléments faisant écho aux principes avancés par le Nouveau Management Public et qui se sont généralisés ces dernières années au sein de l'hôpital public. Les réformes récentes témoignent, pour reprendre l'expression de Claveranne (2003, p. 128), de la volonté politique " de passer du ménagement au management » à l'hô- 
pital. C'est dans ce contexte de « modernisation » que prennent sens les évolutions rencontrées dans l'hôpital public étudié, et plus spécifiquement la mise en place de nouveaux outils d'évaluation des personnels.

\subsection{Moderniser la GRH avec la gestion prévisionnelle des métiers et des compétences}

Si leur objectif premier n'est pas la gestion des personnels hospitaliers, les réformes appliquées ces dernières années à l'hôpital public l'impactent de manière certaine. En matière d'organisation du travail et de GRH au niveau des établissements, elles tendent à remettre en cause le modèle de bureaucratie professionnelle ${ }^{4}$ dont se rapprochent les hôpitaux (Crozet et alii, 2008), au profit de la logique gestionnaire ; cette dernière mettant l'accent sur les problématiques d'efficacité et d'efficience appliquées à la question de l'allocation des ressources, et notamment des ressources humaines.

C'est surtout au début des années 2000 que la GRH des hôpitaux publics a commencé sa "modernisation ", en lien étroit avec le problème de risque de perte des savoir-faire hospitaliers dû aux départs à la retraite. En 2001 a été créé l'Observatoire national des emplois et des métiers de la fonction publique hospitalière, chargé entre autres de l'élaboration du Répertoire des métiers hospitaliers. La GPMC s'est alors développée, en particulier suite à la diffusion d'une circulaire ${ }^{5}$ appelant les établissements de santé à mettre en œuvre, en complément à la gestion statutaire classique, une gestion des métiers et des compétences devant répondre aux risques de perte future de savoir-faire et aux enjeux de professionnalisation des métiers de la santé. Notre travail s'inscrit dans ce contexte de transformation du fonctionnement des hôpitaux publics en mettant l'accent sur la dimension GRH ; ces transformations, comme nous le montrerons, bousculent les cadres de soins. Il alimente à cet égard les travaux récents d'Estryn-Béhar (2008) sur la santé et la satisfaction des soignants au travail. Ces travaux soulignent justement que les responsabilités qui sont confiées aujourd'hui aux cadres de soins, notamment en matière de GRH, peuvent contribuer à accroître leur stress au travail (p. 81).

En matière d'évaluation du travail, dans l'hôpital étudié, des référentiels de compétences avaient été construits il y a plusieurs années pour identifier les compétences requises au sein des emplois d'infirmiers, d'aides soignants et d'agents de services hospitaliers, première étape vers la GPMC. Cependant, ces outils n'étaient pas mobilisés dans le cadre de l'entretien annuel individuel d'évaluation donnant lieu à une "note "; cette note,

4. La bureaucratie professionnelle au sens de Mintzberg est une structure organisationnelle-type caractérisée par une standardisation des qualifications et des savoirs assurant aux " professionnels » une forme d'autonomie dans l'exécution de leur travail.

5. Il s'agit de la circulaire du 9 octobre 2007 relative au financement par le Fonds pour la modernisation des établissements de santé publics et privés (FMESPP) 2007, des projets visant à renforcer la gestion des ressources humaines dans les établissements de santé 
d'une part, déterminait la progression de l'agent au sein de son grade et justifiait, d'autre part, combinée avec les critères d'ancienneté et de présentéisme, une " prime annuelle de service ". Afin de rationaliser la procédure d'évaluation, la DRH a alors cherché à revoir le système existant en instaurant une grille d'évaluation ; trois raisons plus spécifiques expliquent les évolutions à cet égard.

La première est liée à la volonté de la DRH de doter les cadres de soins d'outils visant à rendre l'évaluation plus objective. Avant la mise en place de la grille, des entretiens d'évaluation étaient conduits mais sans outil formalisé a priori. Les cadres de soins prenaient seulement des notes.

La deuxième raison est liée à des obligations légales. Plus spécifiquement, la loi de modernisation de la fonction publique du 2 février 2007, puis le décret du 21 août 2008 relatif à la formation professionnelle tout au long de la vie des agents de la fonction publique hospitalière ont conduit les DRH des hôpitaux publics à intégrer, au sein des évaluations, une partie consacrée au développement des compétences des agents. C'est dans ce cadre que des référentiels de compétences avaient été formalisés initialement.

Enfin, instaurer une grille pour évaluer la qualité du travail des agents constitue une étape importante vers la mise en œuvre d'une politique de GPMC devant permettre notamment de traiter la problématique de l'évolution démographique des personnels et des patients.

\section{Un hôpital public en transformation : focus sur l'évaluation du travail}

Dans l'hôpital étudié, pendant trois ans, les cadres de soins ont été accompagnés par un consultant chargé de développer leurs compétences managériales. C’est au cours de ce suivi qu'ils ont conçu avec lui la grille d'évaluation puis ont été formés à la conduite de l'entretien annuel d'évaluation.

Au niveau formel, la grille d'évaluation utilisée se compose de trois volets. Le premier rend compte d' "objectifs/résultats attendus» dont des indicateurs - si possible quantifiables - sont à spécifier par le cadre, en négociation avec le personnel soignant. Dans le document de synthèse de la formation à l'évaluation suivie par les cadres, l'accent est justement mis sur le management par objectifs. Le responsable d'équipe est décrit comme un "acteur clé dans le développement du management par objectifs" qui "doit intégrer la vision de l'entreprise ${ }^{6}$ afin de la décliner en objectifs opérationnels vers ses équipes».

6. On peut être surpris de voir ici utilisé le terme « entreprise » dans un document utilisé pour former des agents publics. Ceci témoigne des transferts en cours, via les cabinets de consulting, de méthodes utilisées initialement dans les organisations privées et étendues au sein des organisations publiques. Le consultant concepteur du dispositif n'a probablement pas pris le temps de formater le document de présentation du dispositif d'évaluation aux caractéristiques de l'organisation dans laquelle il est intervenu... 
Le deuxième volet de la grille est consacré à l'évaluation des compétences des agents ${ }^{7}$. Cette partie a été articulée avec les référentiels de compétences mentionnés plus haut et décrivant les compétences à détenir au sein des emplois répertoriés par l'hôpital. Il s’agit pour les cadres d'évaluer dans quelle mesure les compétences requises dans l'emploi sont effectivement détenues par l'agent.

Enfin, le dernier volet vise à expliciter les objectifs à atteindre pour la période future. Cette dernière partie aborde par ailleurs les formations à envisager et l'évolution professionnelle de l'agent, compte tenu des résultats de l'évaluation des objectifs et des compétences de la période.

\subsection{La grille d'évaluation : de l'« outil » à la « machine de gestion »}

Alors même qu'ils ont participé à la construction de la grille d'évaluation, les cadres de soins sont très critiques vis-à-vis d'elle. On retrouve ici un fait récurrent de la mise en œuvre des dispositifs d'évaluation du travail : elle fait l'objet de critiques même chez ceux qui ont pourtant participé à son élaboration (Trépo et alii, 2002, p. 51). Deux catégories de comportements sont identifiables : ceux qui estiment que la grille d'évaluation est mal construite ou trop complexe et qui refusent de l'utiliser ; ceux qui s'efforcent d'utiliser la grille même s'ils la trouvent trop compliquée.

Aux yeux des cadres, le nouvel outil n'apparaît pas adapté aux contraintes de leur activité, ni à la fonction qu'ils assurent au sein de l'organisation. Ils ont certes besoin d'évaluer le travail des membres de leur équipe pour s'assurer que le service rendu aux patients est de qualité et ils ne rejettent pas par principe l'utilisation d'une grille d'évaluation. Mais pour eux, la grille formalise de manière non pertinente le travail qu'ils attendent de leurs équipes. Elle leur apparait très complexe et beaucoup trop difficile à faire comprendre aux agents. Deux grandes critiques sont avancées, faisant écho à la distinction faite par Berry (1983) entre les "abrégés du vrai » et les «abrégés du bon " que produit tout outil de gestion.

D'une part, on retrouve, parmi les remarques des cadres, des critiques souvent formulées à l'encontre des outils d'évaluation individualisée qui, dans la manière dont ils ont été construits, peuvent parfois apparaître comme un véritable "défi à la rationalité limitée " (Stankiewicz, 2003, p. 239) : "je ne comprends pas ce quil faut remplir en ce qui concerne 'la fonction' " (encadrement, soins), "certains items ne correspondent pas aux agents" (encadrement, soins), "il faut remplir des cases quion ne comprend pas bien, c'est compliqué " (encadrement, soins). La sophistication de l'outil, la décomposition complexe des types de savoir-faire mobilisés, les termes utilisés paraissent difficiles à saisir. Ne rendant pas

7. À compter de 2011, cette partie donne lieu à un entretien spécifique - l'« entretien de formation " - différent de l'entretien annuel d'évaluation et visant à se caler sur les obligations légales en matière de formation. 8. Après chaque phrase-témoin, nous avons noté le niveau hiérarchique (direction, encadrement ou personnel) et le type de service auquel appartient la personne interrogée (soins ou administration). 
compte correctement, aux yeux des cadres, de la réalité du travail (" abrégés du vrai ») de leurs subordonnés, l'outil déstabilise leur représentation des activités qu’ils doivent faire réaliser à leurs équipes. Aussi sophistiqué que puisse être l'outil d'évaluation, comme l'a bien montré Dejours (1993, p. 29), "le travail effectif ne pourra jamais être intégralement rendu à la visibilité».

L'autre critique émise en direction de la grille renvoie aux limites portées par les « abrégés du bon ", c'est-à-dire des indicateurs - généralement quantitatifs - de résultats à atteindre pour être considéré comme efficace. Cette critique porte sur le volet « management par objectifs ». Le fait d'inciter les cadres à définir des objectifs - chiffrables si possible - aux agents qu'ils dirigent est très mal perçu. Pour eux, il y a une contradiction flagrante entre la fixation d'objectifs mesurables et l'activité réelle de travail des agents de soins auprès des patients. Cette contradiction est accentuée par le fait que les cadres de soins sont souvent eux-mêmes d'anciens infirmièr(e)s et qu'ils connaissent donc bien le travail auprès des patients. On a affaire à un conflit de valeurs patent entre la représentation qu'ont les cadres de soins de ce qu'ils estiment être un "bon » travail de leurs subordonnés et la représentation qu'ils se font du travail que la direction attend d'eux : d'un côté, une logique empathique en direction des patients ; de l'autre, une logique instrumentale au service d'objectifs de performance de l'hôpital : "Ce qui me gêne, c'est qu'on pense que l'hôpital est une entreprise alors qu'on est avec des humains et du coup quand on parle de l'humain, notre direction parle de chiffres. " (encadrement, soins)

Finalement, d' " outil de gestion " mis en place au départ pour faciliter le travail des cadres en matière d'évaluation, la grille d'évaluation est perçue par les cadres comme une " machine de gestion " pour reprendre l'expression de Girin (1983), une " machine » rigide et contraignante à laquelle ils doivent s'adapter, sans forcément en comprendre complètement les rouages; ce que certains psychologues rendent compte à travers la notion $\mathrm{d}$ " activité requise " au sens où tout instrument "constitue pour le sujet un ensemble de contraintes qui simposent à lui et qu'il doit gérer dans la singularité de chacune de ses actions" (Rabardel, 1995, p. 175).

Les cadres de soins les plus critiques refusent d'ailleurs d'utiliser la grille. Ils vivent sa mise en œuvre comme une provocation ou un défi à l'encontre de ce qu'ils estiment être au cœur du métier des infirmièr(e)s ou des aides soignant(e)s qu'ils encadrent, la délivrance de soins , : "Quand je lis 'missions transversales' dans la fiche d'évaluation individuelle que je dois soumettre à une aide-soignante qui ne comprend pas le terme, je trouve que c'est de la provocation." (encadrement, soins) ; "Quand on pond des outils d'évaluation comme ça alors qu'on n'a pas les moyens de garder le personnel contractuel, je me pose des questions!" (encadrement, soins) De manière générale, les cadres de soins sont tiraillés entre la logique gestionnaire imposée par la direction, et ici plus spécifiquement la DRH qui cherche à rationaliser les procédures d'évaluation du travail, et la logique professionnelle tournée vers les patients et les soins. 
Ainsi que le soulignent certains sociologues, le processus de "modernisation " en cours à l'hôpital met les cadres "aux premières loges de la transformation du travail hospitalier. Pendant que certains investissent avec plus ou moins d'hésitations les nouveaux espaces qui leur sont ouverts, d'autres rechignent à assumer le rôle qui leur est dévolu, en particulier parmi les cadres de soins qui restent proches d'une défense des valeurs du service public et du métier d'infirmière " (Divay et Gadéa, 2008, p. 678). Ils jouent en quelque sorte un rôle de " tampon " entre la logique gestionnaire dont ils sont des rouages d'implantation et la réalité du vécu des personnels de soins. Ceci apparaît d'autant plus fortement dans notre cas d'analyse que l'hôpital étudié demeure une petite structure, située dans une ville de petite taille qui favorise une proximité relationnelle forte entre ses agents. À cet égard, la DRH a souligné que, notamment pour cette raison, les cadres étaient selon elle dans une gestion trop « affectuelle » des membres de leurs équipes.

\section{2. "Ce n'est pas comme ça que ça fonctionne entre les hommes "}

La DRH de l'hôpital est très attentive à la question de l'appropriation, par les cadres, des outils qu'elle met en place. C'est d'ailleurs pour cette raison que lorsqu'elle a fait appel aux services d'un consultant, elle n'a pas cherché à intervenir dans la construction de la grille. C'est pourquoi aussi l'instauration de la grille n'est pas perçue comme définitive et devant forcément s'imposer. Au contraire, c'est une première étape, un essai, et la DRH ne s'offusque pas du fait que certains cadres ne l'utilisent pas.

Elle reconnaît d'ailleurs que l'articulation entre les référentiels de compétences et la grille est complexe et questionne même le volet portant sur le management par objectifs : "C'est un peu compliqué, j’aurais aimé quil n'y ait que la grille de compétences." (direction, administration) Face aux critiques émises par les cadres et au jugement de la DRH, il est prévu de revoir la grille d'évaluation.

Pour la DRH, la grille doit d'abord être utile aux cadres et il ne faut pas imposer un outil perçu comme inadapté au contexte de travail et en décalage avec les représentations des utilisateurs. La perspective d'appropriation de l'outil de gestion qui domine ici est « sociopolitique " au sens où, pour reprendre les travaux gestionnaires récents sur l'appropriation des outils de gestion (De Vaujany 2005 ; Grimand, 2006), l'appropriation résulte du jeu des acteurs de l'organisation.

Si la DRH reconnaît la nécessité de développer des outils d'évaluation, elle reste toutefois prudente vis-à-vis de l'idée selon laquelle ces outils sont indispensables pour garantir une objectivité de l'évaluation. Il n'y a pas en effet de croyance ferme en l'objectivité et la neutralité de l'outil. 
Certes, la recherche d'une plus grande objectivité est, comme nous l'avons souligné, mise en avant pour justifier la mise en place de la grille ; toutefois, pour la DRH, l'outil ne garantit pas forcément que l'évaluation soit objective, même si les indicateurs sont correctement formulés, chiffrables ou observables : "Plus j'avance, plus je m’interroge ; ces fameux indicateurs, on en parle beaucoup, on les construit, on les met en place a posteriori pour se justifier; mais je pense que c'est un peu un rêve conceptuel, ça ne se traduit pas complètement dans la réalité; ce n'est pas comme ça que ça fonctionne entre les hommes. " (direction, administration)

De même, au-delà de la question de l'objectivité qui serait induite par l'utilisation de la grille d'évaluation, la DRH exprime des doutes vis-à-vis des outils de gestion qui sont instaurés dans l'objectif d'améliorer les compétences managériales des cadres. Elle argue en effet : "L'outil [d'évaluation] est un guide et il est important, mais on ne peut pas dire qu'il n'existait rien auparavant; ce n'est pas parce qu'on était dans l'oral que rien ne se faisait; on a peut être trop tendance à dire que sans outil, on travaille forcément mal; avec du recul, je pense quil y a des personnes qui ont l'esprit de leader, de manager, qui n'ont pas eu d'outils et qui sont pour autant de bons managers; et il y en a d'autres qui ont eu des outils, et ce n'est pas pour autant qu'ils sont de bons managers" (direction, administration).

Par ailleurs, elle regrette que les nouveaux dispositifs instaurés, sous l'effet des réformes récentes, ne permettent pas d'améliorer les salaires des agents. Cela s'explique en grande partie par le fait que les résultats de l'évaluation n'ont que peu d'effets sur la progression salariale et la carrière des agents. L'évaluation donne certes lieu à une note qui conditionne pour partie l'évolution de l'agent dans son grade et qui détermine une " prime de service " mais l'évolution professionnelle et la rémunération sont prioritairement déterminées par l'ancienneté. La DRH reconnaît se trouver à cet égard dans une position paradoxale ; d'un côté, le ministère incite à la mise en place de règles internes visant à reconnaitre le travail individuel des agents pour influencer leur comportement; de l'autre, les règles de gestion statutaire ne le permettent pas.

Il existe ici un parallèle entre, d'une part, la position des cadres tiraillés entre la logique instrumentale du nouvel outil d'évaluation et la logique professionnelle tournée vers les soins, et, d'autre part, la position de la DRH qui cherche à instaurer des dispositifs " modernes " de GRH, tout en doutant de leur capacité à réellement objectiver l'évaluation et à améliorer les compétences managériales des cadres. 
Comme nous allons le développer maintenant, la source de ces tensions doit beaucoup à la nature des outils de gestion qui, en tant que règles formelles, participent à la construction de rôles sociaux chez les membres de l'organisation, rôles qui conduisent à modifier leur façon d'être et de se représenter leur activité de travail dans le sens de la "philosophie gestionnaire " (Hatchuel et Weil, 1992) de l'outil.

\section{Des outils d'évaluation ambivalents}

La DRH comme les cadres de soins sont bousculés par l'introduction de la nouvelle procédure d'évaluation du travail. La DRH est tiraillée entre deux pôles : d'une part, la pression institutionnelle pour l'instauration de dispositifs « modernes » de GRH et sa volonté personnelle d'outiller les cadres de soins de dispositifs permettant de rationaliser a minima la procédures d'évaluation ; d'autre part, sa conviction personnelle que sur le fond, ce ne sont pas tant les outils de gestion qui sont déterminants que ceux qui les utilisent, en fonction de leur contexte de travail, des contraintes qu' ils rencontrent, des fonctions qu'ils assument.

Quant aux cadres de soins ils sont déstabilisés par la nouvelle grille qu'ils doivent utiliser : ils sont bien conscients qu'il est nécessaire d'évaluer le travail des infirmièr(e)s et aidessoignant(e)s mais ont tendance à critiquer l'outil dont la logique est confuse et l'orientation vers la fixation d'objectifs à atteindre en contradiction avec le travail même de soins.

\subsection{Décisions « intentionnelles » versus décisions " adaptées »}

Selon nous, les tensions évoquées précédemment ont la même source : l'opposition existant entre la rationalité instrumentale portée par la grille d'évaluation nouvellement instaurée et la rationalité contextualisée des individus engagés dans l'action. Les réflexions de James March $(1981,1994)$ sur la prise de décision en organisation, que nous appliquerons ici aux décisions portant sur la valeur attribuée au travail, semblent éclairer les conflits précédemment soulignés.

March fait la distinction entre deux grands types de décisions en organisation : les décisions intentionnelles (willful) et les décisions adaptées (appropriate). Les premières résultent d'une délibération qui est calculée, "basée sur les prévisions portant sur les conséquences à venir des actions courantes" (1981, p. 207).

La mise en place de la nouvelle procédure d'évaluation dans le cas étudié renvoie à cette forme de rationalité que nous qualifierons d'instrumentale au sens que donne Brabet (1993)

9. Les auteurs définissent la "philosophie gestionnaire" d'un outil de gestion comme le "système de concepts qui désigne les objets et les objectifs" (1992, p. 124) de cet outil. 
au modèle de GRH du même nom. Au sein de celui-ci, les comportements des individus sont orientés par les dispositifs mis en place par les directions des ressources humaines, la GRH étant réduite à une affaire de technique. Appliqué à l'évaluation du travail, l'outil est vu dans cette perspective comme un élément neutre et impartial qui facilite le travail des cadres en permettant de lui-même, par sa technicité, de conférer une valeur objective au travail.

L'outil ne ferait que représenter le réel tel qu'il est. Si l'évaluateur est bien formé à son utilisation (identification d'une batterie d'indicateurs de résultats, indicateurs de préférence quantifiables, construction a priori d'un référentiel de compétences, etc.,), la subjectivité du jugement qu'il porte sur le travail à évaluer est gelée, contrôlée à un point tel que dans cette lecture, ce n'est pas l'utilisateur de l'outil qui est producteur du résultat de l'évaluation mais l'outil lui-même. Celui-ci objective une réalité considérée comme donnée et qui existerait indépendamment du regard de celui qui évalue. On retrouve ici ce que Lorino (2002) appelle le statut "représentationniste et computationnel " des outils de gestion selon lequel "l'outil, déterminé par les données réelles de l'action, "reflet" de situations génériques, produirait $[\ldots]$ des conséquences pratiques, par exemple des décisions de la part des acteurs, de façon prédictible et déterministe " (souligné par l'auteur, p. 6). Dans notre cas d'étude, les « décisions de la part des acteurs " permises par l'outil portent sur la valeur du travail.

Ce type de décisions s'oppose aux décisions « adaptées », pour reprendre March. Dans cette perspective, les individus suivent des règles formelles et informelles à partir de leur compréhension personnelle du contexte dans lequel ils interviennent et de la représentation qu'ils se font de leur rôle dans l'organisation (March, 1994, p. 60). Nous parlerons alors de rationalité contextualisée pour rendre compte de cette idée. Ce qui importe ici est l'adéquation du comportement aux règles organisationnelles telles que l'individu les perçoit. La décision est dite "adaptée " au sens où elle s'appuie sur "la compréhension de la nature des choses, des représentations propres [de l'individu] et des images organisationnelles» (March, 1981, p. 221). C'est bien en référence à ces éléments que la DRH émet finalement des doutes quant à la propension de la grille d'évaluation à rendre compte objectivement de la valeur du travail ou de manière plus générale, à la capacité des outils de gestion à pleinement cadrer le comportement des agents. Du côté des cadres de soins, les critiques émises à l'encontre de la grille d'évaluation reposent de même sur la rationalité contextualisée : l'évaluation ne peut résulter de la seule utilisation mécanique ou automatique d'une grille aussi sophistiquée soit-elle. L'outil d'évaluation participe de l'encadrement du jugement émis par les cadres sur la valeur du travail réalisé ; cependant, il le fait dans un contexte de combinaison complexe de règles formelles et informelles au sein duquel, de manière plus générale, chacun assure un rôle socialement construit par ces règles. 
Or justement, les outils de gestion jouent à cet égard une fonction ambivalente. Selon Pezet et Pezet (2010), ils participent à la construction de rôles sociaux en modifiant les comportements des individus et leurs manières d'être. Ils sont donc loin d'être neutres. Pour reprendre les auteurs précédents, ils constituent des "artefacts cognitifs [qui] façonnent les modèles de pensée des acteurs"(p. 78). Les individus intériorisent plus ou moins fortement les idéaux construits par les dispositifs - comme par exemple l'idéal de performance - et se comportent ensuite en référence à eux. Ces idéaux guident les comportements au travail, normalisent les actions et instituent des schèmes de pensée structurant les raisonnements, les interprétations et évaluations des acteurs. Néanmoins, cette intériorisation n'est pas mécanique, automatique. Les individus ne sont pas des automates sans conscience obéissant à la rationalité instrumentale véhiculée par les techniques de gestion. Ils doutent, débattent de cette forme de rationalité, peuvent la contester, orientés en cela par leur rationalité contextualisée. On retrouve ce point de vue chez certains psychologues comme Rabardel (1995) pour qui tout " instrument» - pour reprendre le terme de l'auteur - doit être vu, lorsque l'on étudie son appropriation par les acteurs, comme une "entité mixte» (1995, p. 93) ; il relève en effet à la fois de l'objet et du sujet, c'est-à-dire d'une part de l'artefact matériel ou symbolique dont l'individu fait usage et qui lui permet de réaliser son activité, et d'autre part des schèmes d'utilisation mobilisés individuellement et collectivement et qui sont indispensables à l'utilisation de l'artefact. Dans cette optique, tout instrument n'existe pas indépendamment du sujet. Il n'est pas "donné » a priori mais se construit progressivement et évolue.

Dans notre étude, la nouvelle grille d'évaluation est contestée par les cadres de soins car elle s'appuie sur une convention sur la valeur du travail (Eymard-Duvernay et Marchal, 2000) différente de celle qu'ils suivaient jusqu'à maintenant. L'idéal de performance qu'elle porte heurte en quelque sorte leur éthique professionnelle et leur engagement dans le travail qui reposent sur la primauté accordée à la logique de soins sur celle de la « performance ".

Les cadres sont d'autant plus critiques vis-à-vis de la nouvelle grille qu'à leurs yeux, la logique de soins et la logique de la nouvelle grille d'évaluation sont irréductibles l'une à l'autre. Comme le note Dumond (2003, p. 72) dans une étude sur les conflits de pouvoir à l'hôpital, "les rôles consistant à accueillir, comprendre une souffrance, proposer un soin et l'effectuer d'un côté, et gérer des ressources de l'autre, se réferent à des attitudes pratiques et des valeurs sans commune mesure". Là où la logique de soins se suffit à ellemême, porte en elle sa légitimité et ne sert finalement aucune fin si ce n'est la plus fondamentale - la lutte pour la vie et contre la mort -, la logique instrumentale portée par les réformes récentes prend surtout sens par rapport à des éléments qui lui sont externes, très souvent aujourd'hui de nature économique. 
C'est dans ce contexte que, pour reprendre Estryn-Béhar (2008), "les cadres, y compris ceux de proximité, sont amenés à être des gestionnaires plutôt que des soignants. On constate alors une perte de sens de l'activité, au sein du service, chez le cadre qui se voit transformé en une courroie de transmission, obligé d'assumer au quotidien des décisions qui le mettent parfois mal à l'aise par rapport à son vécu antérieur de soignant" (p. 85). Les cadres de soin sont ainsi tiraillés entre deux pôles. La question est alors de savoir s'il est possible de dépasser les tensions évoquées.

\subsection{Dépasser les tensions?}

Parce qu'elle est centrée sur la question de l'évaluation du travail, l'étude menée ici apparaît en quelque sorte comme une loupe grossissant le conflit qui agite les cadres de soins. L'outil mis en place les incite à évaluer les membres de leur équipe au regard de critères ne correspondant pas à la représentation qu'ils se sont socialement construite $\mathrm{du}$ " bon travail ${ }^{10} \mathrm{~d}^{\prime} \mathrm{un}$ soignant. Or, on attend des cadres de soins qu'ils évaluent la qualité du travail de leurs subordonnés au regard d'un idéal dont ils doutent du bien-fondé. L'instauration de la nouvelle grille d'évaluation les place alors dans une position ambiguë, position que certains travaux sociologiques récents sur la place des cadres dans les hôpitaux publics en " modernisation " ont également bien soulignée. À ce titre, l'enquête menée par Sainsaulieu (2008) sur le collectif soignant a montré que du côté des cadres, le type dominant - qualifié de " cadre animateur " - est traversé par une ambivalence. Elle se traduit d'un côté par un "positivisme " portant sur le goût de faire changer les choses, l'autonomie au travail et le management des agents ; de l'autre côté, par une critique des transformations en cours à l'hôpital se traduisant notamment, selon les cadres, par une pénurie relative de personnel et l'instauration d'une pression financière conduisant finalement à un éloignement des soins.

Comme le notent Divay et Gadéa (2008, p. 687) au sujet des cadres publics «les nouvelles règles du jeu les portent en avant, faisant d'eux la cheville ouvrière du changement, mais c'est parfois à leur corps défendant-ou comme à l'hôpital local, en s'efforçant de s'en préserver-qu'ils le conduisent $"^{11}$.

Du côté de l'État, un rapport a été commandité - Rapport de la mission cadres hospitaliers (De Singly, 2009) - afin d'identifier des voies visant à mieux valoriser et reconnaître la fonction d'encadrement dans les hôpitaux, notamment pour dépasser le type de réactions critiques que notre travail sur l'évaluation souligne. Selon son rédacteur, la formation a un rôle important à jouer à cet égard. Ainsi que le souligne le rapport : " de même que la formation initiale doit préparer les futurs cadres à des responsabilités et des organisations en fortes transformations, la formation continue doit permettre cette adapta-

10. Voir aussi sur ce point Clot (2010) qui parle quant à lui de "travail bien fait».

11.Voir aussi les travaux de Bourret (2006) ou plus récemment Belorgey (2010). 
tion aux cadres en fonction. C'est l'enjeu général et majeur pour tous les cadres hospitaliers" (De Singly, 2009, p. 56).

C'est justement en référence à certaines propositions avancées dans le Rapport de la mission cadres hospitaliers qu'au niveau national, des recommandations ont été faites en 2010 par le ministère de la Santé et des Sports. Elles visaient à organiser, dans tous les hôpitaux publics de France, une "semaine de l'encadrement " afin de répondre à quatre objectifs : "renforcer les cultures communes ", poursuivre les réflexions engagées localement pour "enraciner l'évolution des pratiques managériales ", " accélérer la conception et l'évaluation des projets managériaux " et " contribuer à l'assise d'une véritable communauté de cadres $»^{12}$. Dans l'hôpital étudié, cette "semaine de l'encadrement " s'est notamment traduite par l'invitation d'un sociologue venant témoigner de ses interventions en organisations, par la tenue de plusieurs réunions d'échanges portant par exemple sur les objectifs de l'hôpital, les projets et les réussites en cours.

Dans la perspective managériale dominante et que l'on retrouve dans le Nouveau Management Public, la formation est vue comme une clé d'entrée indispensable pour faire évoluer les agents et adapter leurs comportements aux attentes et exigences des organisations en transformation sous l'effet de réformes. Ceci apparait au niveau des décideurs nationaux, comme nous l'avons précisé précédemment, mais aussi des décideurs locaux à la tête des organisations. Dans le cas spécifique de l'hôpital étudié, rappelons que les cadres de soins ont été sensibilisés à la thématique de l'évaluation lors d'une formation commandée par la DRH et assurée par un consultant. Le suivi des cadres sur plusieurs années par le consultant n'était d'ailleurs pas exclusivement centré sur la thématique de l'évaluation. À côté de l'évaluation, la formation devait aborder deux autres points : d'une part, amener les cadres à homogénéiser leurs pratiques communes; d'autre part, développer leurs compétences d'encadrant. Un des constats de la DRH étant en effet que, comme souligné plus haut, les cadres de soins sont en général insuffisamment inscrits dans une logique managériale et trop proches de leurs équipes, ce qui peut entrainer des pratiques contraires aux contraintes et objectifs de la direction (par exemple, difficultés des cadres à convaincre les membres de leur équipe à changer d'équipe ou à accepter de suivre une formation en dehors de la ville d'implantation de l'hôpital). Pour reprendre Reichard (1998) qui s'est interrogé sur la formation des agents publics aux outils et valeurs nouvelles portés par la "modernisation " de la fonction publique de nombreux pays, le "'nouveau' management public exige de 'nouveaux' managers publics» (p. 178), et la formation répond à ce besoin pour les gouvernants.

12. Selon les termes du document envoyé pour mise en œuvre par la Direction générale de l'offre de soin du ministère aux directeurs des établissements publics de santé. 
Or pour l'auteur, le succès des gouvernements à développer, chez les cadres publics, les compétences requises par le Nouveau Management Public, repose sur la manière dont les formations vont agir sur la pensée (thinking) des agents. Selon Reichard, les expériences menées dans différents pays font ressortir deux grands choix contradictoires : "quand les formés ont suivi une formation radicale très orientée "Nouveau Management Public", ils courent le risque d'être isolés ou rejetés du système administratif en place. S'ils choisissent d'assimiler eux-mêmes la culture liée au Nouveau Management Public, ils s'insèreront mieux dans le système. Mais cette assimilation pose des problèmes d'adaptation aux règles bureaucratiques et aux “anciens” comportements" (p. 181). Tout l'enjeu reposerait alors, selon l'auteur, sur le choix du bon moment (readiness) auquel les innovations sont mises en œuvre.

Selon cette hypothèse, l'échec de l'instauration de nouvelles procédures d'évaluation du travail des agents de soins dans l'hôpital étudié serait dû à un mauvais timing. Les cadres de soins ne seraient pas encore prêts à voir s'instaurer des dispositifs d'évaluation centrés sur la prise en compte des performances et compétences des individus. Or au regard de notre grille de lecture théorique, ce n'est pas tant le choix du «bon moment " qui explique l'attitude des cadres que l'opposition quasi-irréductible à leurs yeux entre la rationalité instrumentale portée par le nouvel outil d'évaluation et la rationalité contextualisée qui oriente leur action au quotidien. S’il n'est pas simple de trouver des solutions immédiates et opérationnelles permettant de dépasser cette opposition, risquons-nous toutefois, pour finir, à en avancer trois, alimentées par les réflexions menées jusqu'à maintenant : élaborer des outils d'évaluation simples ; reconnaître les tensions pour accepter la complexité du réel ; concevoir une formation plus englobante.

D'abord, en matière de construction de grilles d'évaluation basées sur des référentiels de compétences tout comme sur la spécification d'objectifs à atteindre, il faut éviter de construire des outils assimilés par les utilisateurs à des " usines à gaz " ou, comme souligné plus haut, à des "machines de gestion " à la logique desquelles les individus doivent se soumettre sans trop en comprendre le sens alors qu'au départ, les outils ont été conçus pour faciliter certaines de leurs tâches. La probabilité est sinon grande de créer des dispositifs dans lesquels évaluateurs comme évalués ne retrouvent pas l'activité réelle de travail qu'ils font exécuter ou exécutent. Un dispositif trop complexe peut déstabiliser les représentations des acteurs et risque d'être rejeté à terme. 
Ensuite, dans la perspective de déconstruction du modèle instrumental de GRH initiée par exemple en France par des auteurs comme Brabet (1993), il nous paraît nécessaire d'amener les acteurs de l'évaluation à reconnaître les paradoxes et les tensions qu'ils traversent et qui sont liés au fait qu'en tant que membres d'une organisation, ils sont sans cesse dans un rapport jamais tranché entre la position de sujet et d'objet : "dans la place de "sujets organisationnels", ils sont créateurs d'objectifs et de fonctionnements collectifs; dans la position où ils deviennent "objets", ils agissent comme des instruments asservis à ces objectifs et ces fonctionnements" (Louart et alii, 1993, p. 179). Reconnaître que toute organisation est un lieu de tensions constitue une condition à l'acceptation de la complexité du réel. Les membres des équipes RH ont ici une responsabilité importante d'accompagnement des individus, évaluateurs comme évalués.

Enfin, s'il nous paraît effectivement indispensable de former les évaluateurs à l'utilisation des outils qu'ils mobilisent et de manière plus générale leur permettre de développer les compétences requises par l'organisation, la formation ne doit pas avoir qu'une dimension instrumentale au service de la seule organisation. Les individus doivent aussi être conscients des limites des outils de gestion qu'ils utilisent et du fait que ceux-ci ne résoudront pas de manière mécanique tous les problèmes qu'ils rencontrent. La formation doit jouer à cet égard un rôle important.

Au-delà de la conception managériale dominante de la formation comme moyen d'adaptation des individus aux organisations, nous plaidons ici pour une approche critique de la formation non pas au sens négatif - c'est-à-dire contre la perspective instrumentale - mais positif. Dans cette voie, au-delà des compétences utiles à l'organisation, la formation doit offrir aux individus des clefs de compréhension et d'analyse de leur situation de travail spécifique et de leur organisation d'appartenance mais aussi de ce qui se passe dans les autres organisations voire au niveau sociétal. L'objectif est de mettre à leur disposition des grilles d'analyse leur permettant de questionner leur pratique, de les resituer dans un cadre plus large afin que chacun développe sa propre opinion sur les évolutions en cours. Ainsi que le notent Pezet et Pezet (2010), la formation des individus est un enjeu capital et «le management est devenu tellement hégémonique qu'il est urgent de [les] former à ses techniques mais aussi à ses limites, à ses sous-jacents, à ses effets. Les salariés en formation continue sont demandeurs d'une telle évolution" (p. 128).

Concrètement, on pourrait adosser les formations sur l'utilisation des outils à des modules plus généraux puisant dans les apports des sciences sociales pour montrer toute la complexité des phénomènes humains et sociaux dans les organisations. Nous rejoignons sur ce point les propositions de Le Goff (1996) qui insiste sur l'importance des formations visant à développer la culture générale des individus en formation initiale comme continue. Il suggère en outre la mise en place de modules de formation du type " approche pluridisciplinaire du travail » ou " connaissance de l'environnement ", modules amenant les individus à prendre du recul vis-à-vis des problèmes auxquels ils font face dans l'organisation et des outils qu'ils mobilisent. L'auteur évoque alors le "paradoxe de la culture générale " 
selon lequel «c'est à la condition d'opérer un décentrement et un recul réflexif par rapport à la pratique quelle [la culture générale] peut éclairer cette pratique et permettre de mieux la mener " (Le Goff, 1996, p. 131). Dans notre optique, cette conception de la formation est plus large et englobante que la conception dominante instrumentale. Elle peut contribuer à développer, chez les individus, un intérêt pour des problématiques nouvelles pour eux. Il s'agit d'aller au-delà des problèmes spécifiques qu'ils rencontrent au quotidien pour mieux les situer et comprendre les enjeux qui se jouent autour d'eux. Potentiellement, elle est porteuse de plus de participation et de débats démocratiques sur les orientations et directions prises et à prendre dans l'organisation, voire au-delà. Potentiellement encore, elle constitue un moyen pour les individus de discuter les évolutions voulues par les dirigeants et de proposer de nouvelles voies. Elle ouvre à une gestion moins unilatérale des organisations.

\section{Conclusion}

Les résultats présentés ici reposent sur des données recueillies au sein d'un établissement unique, ce qui est naturellement insuffisant pour qu'ils soient généralisés. Des études monographiques futures devront fournir d'autres repères venant confirmer ou non les enseignements tirés de ce travail.

Néanmoins, au-delà de cette limite inhérente aux recherches monographiques sur cas unique, notre étude permet de nourrir les réflexions menées en gestion (Berry, 1983 ; Moisdon 1997 ; Gilbert 1998), en sociologie (Maugeri, 2001 ; Boussard et Maugeri, 2003) et en psychologie (Rabardel, 1995, 2005) sur le rôle des " outils de gestion " au sein des organisations. Elle alimente les débats en cours sur la " managérialisation " des organisations publiques appliquée à la thématique de l'évaluation du travail très discutée aujourd'hui.

Pour reprendre Moisdon (1997), les outils de gestion ont une double nature : l'une tournée vers la connaissance et visant à rendre compte de la réalité organisationnelle à un moment donné ; l'autre tournée vers la "conformation ", c'est-à-dire qu'ils spécifient formellement des comportements à adopter. C'est surtout sur cette seconde dimension que notre travail a mis l'accent, en se focalisant sur les outils d'évaluation du travail. En prescrivant un idéal sur ce que doit être un «bon travail ", ces outils œuvrent à une normalisation des comportements attendus par ceux qui gouvernent les organisations et par délégation, toute la ligne hiérarchique. Les démarches d'évaluation du travail apparaissent alors comme un rappel des exigences avancées par ceux qui dirigent, exigences souvent masquées derrière des objectifs présentés comme rationnels et socialement neutres (Dumond, 2006). En cela, les outils d'évaluation participent, pour reprendre la terminologie foucaldienne, de la "gouvernementalité » des organisations et méritent donc toute l'attention des chercheurs. 
Cependant, il serait erroné de croire que les dispositifs d'évaluation du travail possèdent intrinsèquement le pouvoir de normaliser complètement, au regard des attentes de ceux qui dirigent ou réforment, les attitudes et les manières de penser des acteurs. La rationalité contextualisée sur laquelle les individus s'appuient et qui forge leur identité au travail module la capacité des outils à orienter les comportements dans le sens prévu au départ. Elle peut même la contrecarrer et participer, comme nous l'avons vu dans l'hôpital public étudié, de sa remise en cause. Pour les directions et les réformateurs œuvrant pour le changement, la formation des individus apparait alors souvent comme un moyen de faire évoluer les compétences des agents mais aussi leurs représentations. Ainsi que le note March (1994, pp. 71-72), "La plupart des formations formelles et informelles qui ont lieu dans les organisations concourent à défnir des identités, à catégoriser des situations et à appliquer les règles appropriées. Cela produit des modèles qui sont des exemples de bons (proper) comportements ". À cet égard, du point de vue des formations " formelles " pour reprendre March, nous avons plaidé pour une conception moins instrumentale et plus critique de la formation que celle véhiculée aujourd'hui par le Nouveau Management Public. Selon nous en effet, le dépassement des tensions engendrées par les réformes actuelles et ressenties par certains agents publics peut s'opérer en fournissant justement à ceux-ci les moyens de questionner les évolutions voulues par les dirigeants ou gouvernants, à partir de formations à la culture générale puisant dans les travaux menées en sciences sociales sur les organisations et leur évolution. Ce recul réflexif offre, nous semble-t-il, un moyen de promouvoir une meilleure conception et appropriation des outils de gestion.

Par leur dimension technique et leur sophistication, les outils d'évaluation du travail peuvent paraître déconcertants. Comme nous l'avons montré, la grille d'évaluation du travail nouvellement instaurée dans l'hôpital étudié apparaît d'une grande complexité pour les cadres de soins évaluateurs des infirmièr(e)s et aides soignant(e)s et en décalage avec l'activité réelle de travail, à tel point que certains cadres refusent de l'utiliser. Pour ces raisons, ces outils ne doivent pas rester aux seules mains des " experts " qui en sont concepteurs, tant l'enjeu principal auquel ils sont liés - la question de la valeur du travail est essentiel. Le rôle fondamental de ces outils se révèle dans le chassé-croisé actuel entre organisations privées et organisations publiques : d'un côté, plusieurs grandes organisations privées aimeraient voir se développer chez leurs salariés des valeurs en lien fort avec l'éthique du service public - comme par exemple le bien commun ou l'intérêt général. Elles formalisent leurs attentes à cet égard au sein de chartes dont les éléments peuvent être repris dans les outils d'évaluation du travail (Salmon, 2007) ; de l'autre côté, les organisations publiques revoient leur manière d'évaluer le travail des agents en cherchant à faire en sorte qu'ils intègrent, dans leurs comportements et attitudes, des valeurs faisant écho au monde marchand, comme la performance et l'efficience. Or concernant les organisations publiques, la tendance actuelle, outillée par les règles formelles d'évaluation du travail, pose selon nous question. Comme nous l'avons vu dans notre étude de cas, les cadres de soins ont tendance à contester la nouvelle forme d'évaluation car elle déstabilise leur représentation du "bon travail » réalisé par les infirmièr(e)s et aide-soignant(e)s. L'idéal porté 
par les nouvelles règles d'évaluation apparaît contradictoire avec leur éthique professionnelle et le fondement de leur engagement et de celui de leurs subordonnés pour le travail de soins ; ce qui est éminemment problématique pour une organisation dont la fonction première est la délivrance de soins. On peut certes être plus ou moins " efficace " lorsque l'on délivre un soin. Mais il semble toutefois fondamental de veiller à ce que l' " efficacité " en question, en grande partie spécifiée par les outils d'évaluation, ne soit pas trop unilatéralement définie ; et ce à plus forte raison lorsque le service rendu est un service public.

\section{Bibliographie}

Actes de la recherche en sciences sociales (2011), L'évaluation: contextes et pratiques, $\mathrm{n}^{\circ} 189$.

Aron R. (1981), "Quelques remarques sur la compréhension et l'explication ", Revue européenne des sciences sociales, vol. 19, $\mathrm{n}^{\circ}$ 54-55, pp. 71-82.

Bartoli A. (2009), Management dans les organisations publiques, 3ème ed., Dunod, Paris.

Baudry B. et Dubrion B. (2005), "Quel modèle d'évaluation du travail ? ", Travail et Emploi, n' 104, pp. 7-18.

Belorgey N. (2010), L’hôpital sous pression. Enquête sur le "nouveau management public", La Découverte, Paris.

Berry M. (1983), Une technologie invisible? L'impact des instruments de gestion sur l'évolution des systèmes humains, Rapport du CRG, École Polytechnique.

Bourret P. (2006), Les cadres de santé à l'hôpital, Seli Arslan, Paris.

Boussard V. et Maugeri S. (dir.) (2003), Du politique dans les organisations, L'Harmattan, Paris.

Brabet J. (1993), "La gestion des ressources humaines en trois modèles ", in Brabet J. (coord.), Repenser la gestion des ressources humaines?, Économica, Paris, pp. 69-141.

Cahiers Internationaux de Sociologie (2010), "Ce qu'évaluer voudrait dire », n 128-129.

Claveranne J.-P. (2003), "L'hôpital en chantier : du ménagement au management », Revue Française de Gestion, n 146 , pp. 125-129.

Clot Y. (2010), Le travail à cour. Pour en finir avec les risques psychosociaux, La Découverte, Paris.

Couty E., Kouchner C., Laude A. et Tabuteau D. (2009), La loi HPST, regard sur la réforme du système de santé, Presses de l'EHESP Rennes.

Crozet P., Kaaniche A. et Lienard J. (2008), « Nouvelles gouvernance à l'hôpital : recomposition de l'organisation et gestion des ressources humaines ", Politiques et Management Public, vol. 26, n $^{\circ}$, pp. 31-51. 
Cueille S. et Renucci A. (2000), « Responsabilisation des acteurs dans les hôpitaux publics français et management : analyse des dernières réformes juridiques ", Politiques et Management Public, vol. 18, $\mathrm{n}^{\circ}$ 2, pp. 43-68.

De Singly C. (2009), Rapport de la Mission Cadres Hospitaliers, ministère de la Santé et des Sports.

De Vaujany F.-X. (dir.) (2005), De la conception à l'usage. Vers un management de l'appropriation des outils de gestion, EMS, Paris.

Dejours C. (2003), L'évaluation du travail à l'épreuve du réel, Inra ed. Paris.

Divay S. et Gadea C. (2008), «Les cadres de santé face à la logique managériale », Revue française d'administration publique, $\mathrm{n}^{\circ} 128$, pp. 677-687.

Dumond J.-P. (2003), « Les conflits de pouvoir à l'hôpital », Sève, nº 1, pp. 71-81.

Dumond J.-P. (2006), «L'appréciation des personnels. Évaluer n’est pas expliciter », Revue Française de Gestion, n 162, pp. 21-34.

Estryn-Behar M. (2008), Santé et satisfaction des soignants au travail en France et en Europe, Presses de l'EHESP, Rennes.

Eymard-Duvernay F. et Marchal E. (2000), "Qui calcule trop finit par déraisonner : les experts du marché du travail ", Sociologie du Travail, vol. 42, pp. 411-432.

Gilbert P. (1998), L'instrumentation de gestion, Économica, Paris.

Girin J. (1983), "Les machines de gestion ", in Berry M. (ed.), Le rôle des outils de gestion dans l'évolution des systèmes sociaux complexes, Ch.V-1, Ecole polytechnique, CRG, p. $1-4$.

Grimand A. (2006) (dir.) (2006), L'appropriation des outils de gestion. Vers de nouvelles perspectives théoriques?, Publications de l'Université de Saint Etienne, Saint Etienne.

Hatchuel A. et Weil B. (1992), L'expert et le système, Économica, Paris.

Le Goff J.-P. (1996), Les illusions du management, La Découverte, Paris.

Lorino P. (2002), Vers une théorie pragmatique et sémiotique des outils appliqués aux instruments de gestion, WP ESSEC, DR02015.

Louart P. et alii (1993), "Les champs de tension en gestion des ressources humaines ", in Brabet J. (coord.), Repenser la gestion des ressources humaines ?, Économica, Paris, pp. 165-212.

March J. (1981), "Decision Making Perspective”, in Van de Ven A. et Joyce W. (eds), Perspectives on organization design and behaviour, Wiley, New York, pp. 205-244.

March J. (1994), A Primer on Decision Making, Free Press, New York.

Maugeri S. (2001), Délit de gestion, La Dispute, Paris. 
Miles M. et Huberman A. (1991), Analyse des données qualitatives, De Boeck, Paris.

Minvielle E. (2003), «De l'usage de concepts gestionnaires dans le champ de la santé. Le cas de la qualité hospitalière ", Revue Française de Gestion, n 146, p. 167-189.

Moisdon J.-C. (dir.) (1997), Du mode d'existence des outils de gestion, Seli Arslan, Paris.

Moisdon, J.-C. et Tonneau D. (1999), La démarche gestionnaire à l’hôpital, T.1, Seli Arslan, Paris.

Nouvelle revue de psychosociologie (2009), "La passion évaluative ", $\mathrm{n}^{\circ} 8$.

Pezet A. et Pezet E. (2010), La société managériale. Essai sur les nanotechnologies de l'économique et du social, La ville brûle, Paris.

Rabardel P. (1995), Les hommes et les technologies. Approche cognitive des instruments contemporains, A. Colin, Paris.

Rabardel P. (2005), "Instrument, activité et développement du pouvoir agir », in Teulier R. et Lorino P. (dir.), Entre connaissance et organisation : l'action collective, La Découverte, Paris, pp. 251-265.

Reichard C. (1998), "Education and Training for New Public Management", International Public Management Journal, vol. 1, n² 2, pp. 177-184.

Sainseaulieu I. (2008), "Le collectif soignant: mythe ou réalité ? ", Revue Française d'Administration Publique, $\mathrm{n}^{\circ}$ 128, pp. 665-675.

Salmon A. (2007), La tentation éthique du capitalisme, La découverte, Paris.

Savall H. et Zardet V. (2004), Recherche en sciences de gestion : approche qualimétrique, Économica, Paris.

Stankiewicz F. (2003), «La démarche compétence comme solution à un problème d'incitation - le point de vue d'un économiste ", in Dupray A., Guitton C., Monchatre S., Réfléchir la compétence, Octarès Editions, Toulouse, pp. 227-241.

Travailler (2011), "Évaluation du travail et santé mentale ", $\mathrm{n}^{\circ} 25$.

Trepo G., Estellat N. et Oiry E. (2002), L’appréciation du personnel, mirage ou oasis ?, Editions d'Organisation, Paris.

Van Maanen J. (1979), "Reclaiming Qualitative Methods for Organizational Research », Administrative Science Quarterly, vol. 24, n 4, p. 520-526.

Vatin F., Caille A. et Favereau O. (2010), «Réflexions croisées sur la mesure et l'incertitude ", Revue Française de Gestion, n² 203, p. 163-181.

Wacheux F. (1996), Méthodes qualitatives et recherche en gestion, Économica, Paris. 


\section{L'ORIENTATION SCOLAIRE ET PROFESSIONNELLE}

Revue de l'Institut national d'étude du travail et d'orientation professionnelle

\section{Numéro 42/1 (mars 2013)}

Hugues Draelants

Le rôle de l'établissement d'enseignement secondaire dans la construction des aspirations d'études supérieures

Mélissa Arneton, Christine Bocéran \& André Flieller

Les performances en mathématiques des élèves des départements d'Outre-

Mer

Cathy Perret

Réussir son entrée à l'université dans des filières scientifiques

Valérie Capdevielle-Mougnibas, Yves Prêteur \& Cécile Favreau

Rapport au savoir et construction des parcours de formation au sein de la formation professionnelle initiale de niveau $\mathrm{V}$

\section{Benoit Fromage}

L'Épreuve des Trois Arbres, présentation d'un outil d'aide à l'orientation selon le « life designing »

\section{Abonnement 2013 (4 numéros par an)}

France : 112 Euros, Étranger : 118 Euros, Vente au numéro : 45 Euros

OSP - Service abonnement

41 rue Gay-Lussac - 75005 Paris

0144107833 - inetop-osp@cnam.fr 